The means for the two series were pregnant 119 , non-pregnant 127. Estimation of the significance of the difference between these means was made ${ }^{3}$ giving $t=6.114$ for $n=30$. The limit of the published tables is $P=0.001, t=3.646$ for $n=30$. The difference is therefore highly significant, $P$ being less than 0.001 , and the definite statement may be made that there is a decrease in the serum chloride concentration in mice during pregnancy. There is no correlation between the serum chloride level and litter size.

The explanation of this decrease is at present obscure. There are many facts and theories which may be relevant, the following being considered to be the most obvious: the increase in salt appetite during pregnancy ; the relation of the ovaries to salt metabolism ; the effect of pregnancy on adrenalectomized animals ${ }^{7}$ : the beneficial effect of restriction of salt intake on the toxæmia of pregnancy is well known; the theory that the fotal adrenal cortices are active ${ }^{8}$; the hyperpigmentation of normal pregnancy; the influence of sodium chloride on melanin formation ${ }^{\circ}$. A working hypothesis has been formed from these and other statements; but there is in sufficient critical evidence to justify its publication at this stage. An attempt is being made to obtain the necessary evidence.

The work has been carried out with the aid of a grant for the Leverhulme Research Fellowship.

Medical Services,

Ministry of Pensions,

Norcross, Blackpool. Feb. 11 .

1 Lea, A. J., Nature, 158, 556 (1946).

- Grüneberg, H., J. Hered., 34, 89 (1943).

"Fisher, R. A., "Statistical Methods for Research Workers", 128 (Oliver and Boyd, 1938).

- Fisher, R. A., and Yates, F., "Statistical Tables for Biological, Agricuitural and Medical Research", 30 (Oliver and Boyd, 1943). - Richter, C. P., and Barelare, B., Endocrin., 23, 15 (1938).

- Thorn, G. W., and Harrop, G. A., Science, 86, 40 (1937).

'Stewart, G. N., and Rogoff, J. M., Proc. Soc. Exp. Biol. and Med., 23, 190 (1927).

- Parks, T. J., Med. Rec., 156, 355 (1943).

- Lea, A. J., Nature, 155, 428 (1945).

\section{Microdetermination of Copper in Biological Substances}

IN the course of a series of investigations by $F$. Buscarons and E. Loriente ${ }^{1,2}$ on analytical applications of $3,3^{\prime}, 4,4^{\prime}$-diamine-3,3'-dimethoxy-biphenilo (o-dianisidin), these authors have shown that this substance in certain conditions possesses extreme sensitivity for cupric copper. We have used this reactant for colorimetric determinations of copper in the blood and in other biological substances.

The mechanism of the reaction is based in the green coloration that occurs when solutions containing cupric copper are treated with an acetic solution of orthodianisidin and afterwards with a slight excess of sulphocyanide. This coloration tends to change to yellow when the concentrations of copper are around $1 / 10^{6}$; if the concentration is greater than $1 / 5.10^{5}$ much blue precipitate is produced.

For the determination of the copper in small quantities it is necessary to purify all the reactants and use twice-distilled water in a glass apparatus; glass or porcelain burners should be used, because brass ones easily pollute the atmosphere. We use the wet method of analysis because by the dry method volatile copper products are easily formed. Nitro-perchloric acid is used, warming until the liquid is decolorized and there are plenty of fumes of perchloric acid. To eliminate the ferric iron which with the dianisidin gives a red coloration, we precipitate the copper as sulphide; and to help this precipita. tion, and to make the precipitate easier to handle, before passing the current of hydrogen sulphide through the solution we add a small quantity of 4 per cent lead acetate solution (dianisidin gives no colour with lead, at least in the conditions in which we work).

The precipitate is centrifuged and washed; it is then dissolved in some drops of nitric acid and evaporated to dryness on a bath at $150^{\circ}$ in order to eliminate the nitric acid. The residue is dissolved with water, and to this aqueous solution we add a few drops of a freshly prepared and colourless acetic solution of dianisidin, and then ammonium sulpho. cyanide in a light excess, plus one third of the volume of the solution of ethyl alcohol. A green coloration is produced if copper is present; with quantities of copper less than a gamma per c.c. the colour turns yellow in 10-20 min. and remains at least two hours. This colour is compared colorimetrically with that produced by a standard solution of copper with a concentration similar to that suspected in the sample to be analysed and treated in the same way.

$$
\text { E. LORIENTE }
$$

Chemical Analysis Laboratory of E.P.I., Valladolid.

Internal Medical Clinic,

University of Valladolid.

\section{J. CASAS}

${ }^{1}$ Buscarons, F., and Lorfente, E., Anal. Fris. y Quim., 40, 1312 (1944); 41, 249 (1945); 41, 257 (1945).

${ }^{2}$ Loriente, E., doctoral thesis.

\section{Vicinal Action in Steroids}

THE optical rotatory powers of steroidal compounds can be correlated by means of the method of molecular rotation differences, and such correlation has been shown to be of value in solving certain types of structural problem ${ }^{1}$. In some cases anomalies of optical rotatory power occur which cannot be ascribed to trivial causes, but which must be due to disturbing factors generally given the name 'vicinal action'.

It has been assumed that the forces responsible for vicinal action decrease with distance and must become inoperative beyond a certain point ${ }^{2}$; the magnitude of vicinal action may be represented by the expression $\Delta \Delta$ value as used in Tables 1 and 2 . The fused-ring structure peculiar to steroids, together with other factors, makes these compounds particularly suited to the study of the variation of vicinal action with distance, and accordingly selected examples have been examined from this point of view. Tables 1 and 2 refer to a number of compounds which have been prepared in a state of special purity and for which rotation measurements have been made in chloroform solution for the sodium $D$ line. Specific rotations have been rounded off to the nearest degree in calculating the molecular rotations so that, in the absence of vicinal action, $\Delta_{1}, \Delta_{2}$ and $\Delta_{3}$ values (see Tables 1 and 2) should agree with the corresponding standard values for cholesterol and cholestanol to at best \pm 4 . 